\title{
Rechallenge with Lenvatinib after Refractoriness to Initial Lenvatinib Followed by Sorafenib in a Patient with Metastatic Papillary Thyroid Carcinoma
}

\author{
Masaki Takinami Tomoya Yokota \\ Division of Gastrointestinal Oncology, Shizuoka Cancer Center, Shizuoka, Japan
}

\author{
Keywords \\ Differentiated thyroid cancer - Tyrosine kinase inhibitor $\cdot$ Rechallenge $\cdot$ Resensitization . \\ Drug resistance
}

\begin{abstract}
Two tyrosine kinase inhibitors, lenvatinib and sorafenib, are available systemic therapies for patients with metastatic differentiated thyroid carcinoma. However, the treatment options for carcinoma refractory to both lenvatinib and sorafenib are limited. Here, we present a case of metastatic papillary thyroid carcinoma that showed resensitization to rechallenge with lenvatinib. A 72-year-old woman who had been diagnosed with papillary thyroid carcinoma with multiple lymph node and lung metastases progressed with the emergence of subcutaneous metastasis after 3 years of response to initial lenvatinib. Five months after switching to sorafenib, the tumor was enlarged with increased contrast enhancement on computed tomography, suggesting progressive disease. Three months after the reintroduction of lenvatinib, marked tumor shrinkage and a decrease in its contrast enhancement were seen. Rechallenge with lenvatinib showed tumor resensitization. Therefore, rechallenge with lenvatinib may be a treatment option in patients who have experienced progressive disease after initial response to lenvatinib and subsequent sorafenib.

(c) 2020 The Author(s)

Published by S. Karger AG, Basel
\end{abstract}

\section{Introduction}

Standard treatment for metastatic differentiated thyroid carcinoma (DTC), including papillary or follicular thyroid carcinoma, is thyroidectomy followed by radioactive iodine (RAI) ablation [1]. In patients with metastatic, RAI-resistant DTC, two tyrosine kinase inhibitors (TKIs) are approved as systemic treatments by both the European Society for Medical 
A
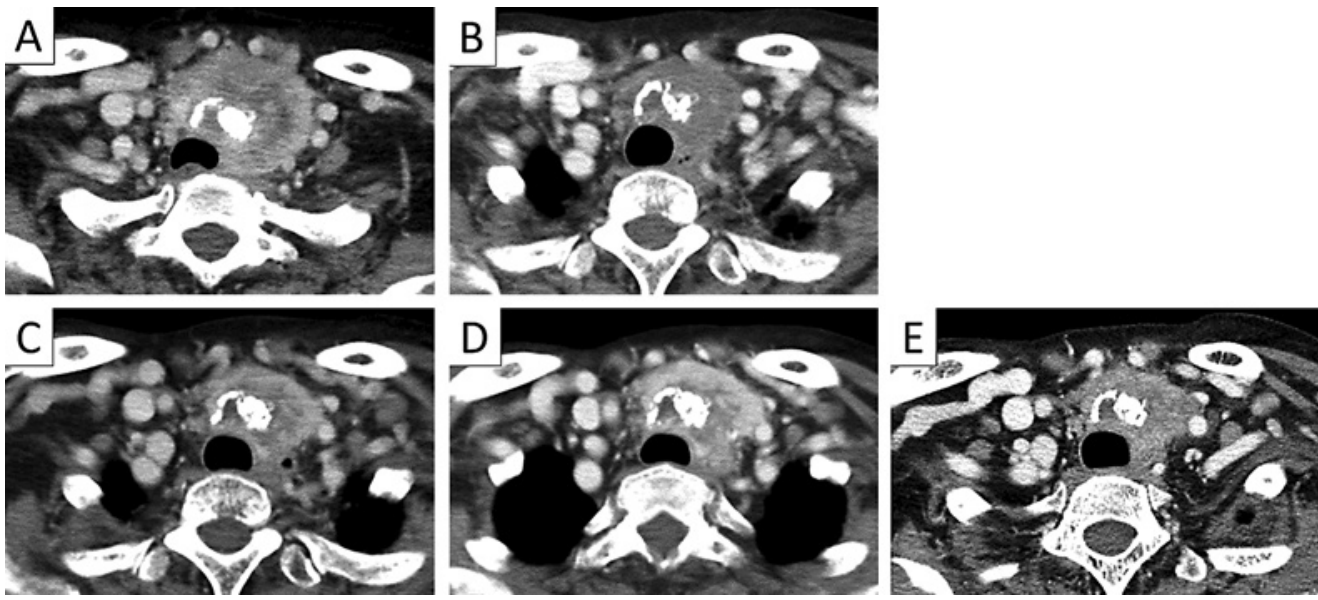

Fig. 1. Primary lesion. A Before treatment. B Three months after the start of initial lenvatinib. C Three years after the start of initial lenvatinib. D Five months after the start of sorafenib. E Three months after the start of rechallenge with lenvatinib.

Oncology (ESMO) and the American Thyroid Association (ATA) [2, 3]. However, there are no recommended treatments for DTC that is refractory to both targeted therapies.

We report a case of papillary thyroid carcinoma that showed resensitization by rechallenge with lenvatinib following progressive disease after both initial lenvatinib and sorafenib.

\section{Case Report}

A 72-year-old woman presented with a 1-month history of hoarseness and anterior swelling at the neck. She had a past history of hypertension treated with valsartan. Physical examination revealed an immovable left-sided thyroid mass. Ultrasonography showed an irregularly shaped tumor with calcifications, and fine needle aspiration cytology revealed papillary thyroid carcinoma. Computed tomography (CT) showed a 5.5-cm, heterogeneously enhanced thyroid tumor that constricted the trachea (Fig. 1A) and multiple lymph node and lung metastases. Because the tumor was judged inoperable due to severe invasion into the sternum manubrium, lenvatinib was immediately initiated at $24 \mathrm{mg} /$ day. Figure 2 shows the treatment course and serum levels of thyroglobulin (Tg). After 3 months, partial response was achieved with reductions in tracheal constriction and contrast enhancement on a CT scan (Fig. 1B), and with a decrease in serum Tg level. During 3 years of response to lenvatinib, a dose reduction of lenvatinib to $4 \mathrm{mg}$ every other day was required because of hypertension and proteinuria. Levothyroxine was simultaneously given to suppress serum thyroid-stimulating hormone levels.

Then, a new lesion emerged as a subcutaneous nodule on the head (Fig. 3). A surgically resected specimen of this nodule histologically revealed metastasis of thyroid carcinoma. Furthermore, a CT scan demonstrated an increase in marginal enhancement in the primary lesion (Fig. 1C). These findings suggested that the lesions were refractory to initial lenvatinib. Treatment was switched to sorafenib at $400 \mathrm{mg} /$ day. After 5 months, the tumor showed greater uptake of contrast agent and enlargement, and the serum $\mathrm{Tg}$ level increased remarkably, suggesting progressive disease (Fig. 1D).

Because the tumor was considered to be refractory to both lenvatinib and sorafenib, the patient was subjected to a FoundationOne ${ }^{\circledR} \mathrm{CDX}$ comprehensive cancer genomic profiling test. 


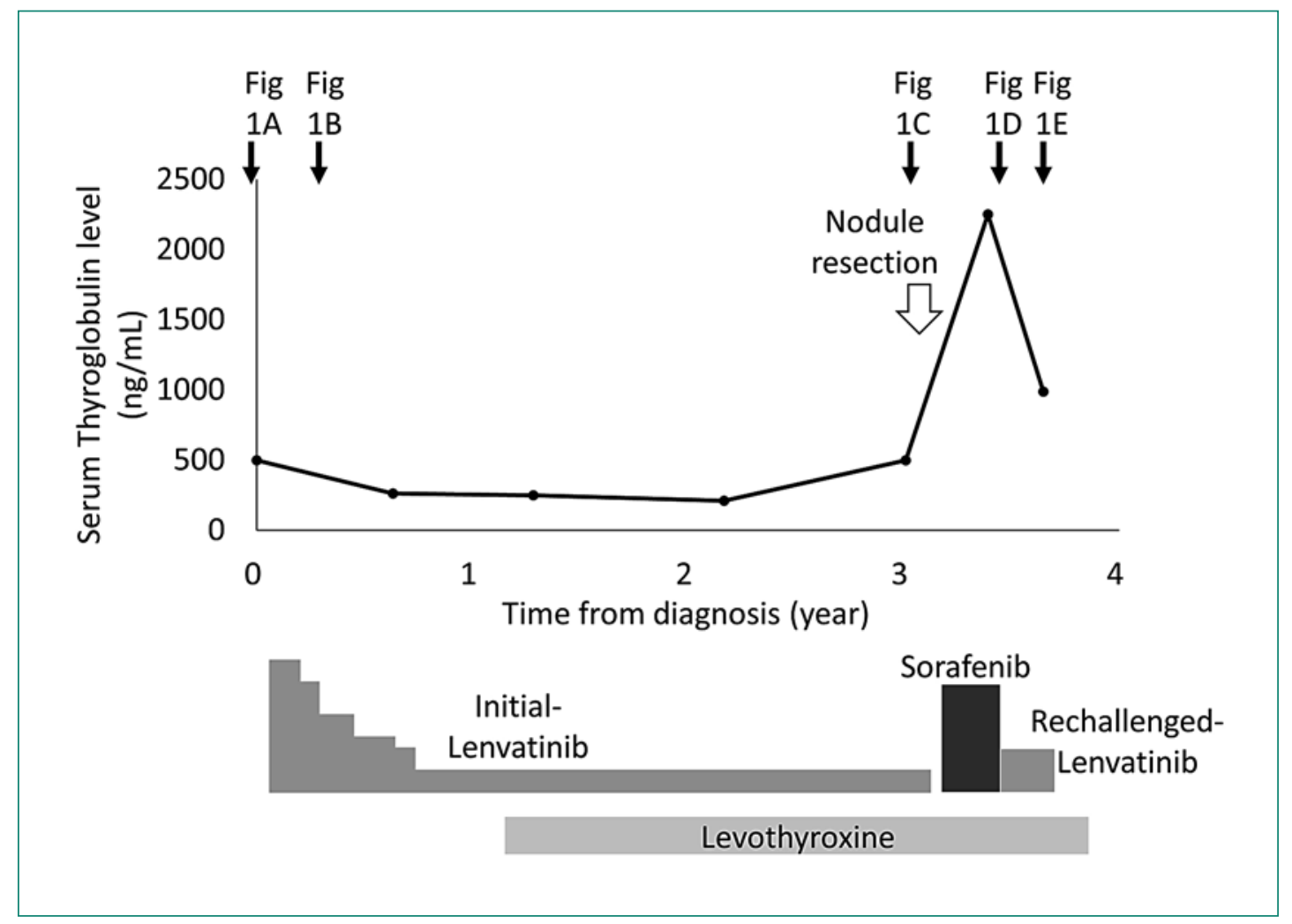

Fig. 2. Treatment course and serum thyroglobulin level. The time points of the CT scans are shown by black arrows (referring to the respective panels in Fig. 1).

Fig. 3. A subepithelial nodule emerged during treatment with initial lenvatinib and was histologically revealed as metastasis of the papillary thyroid carcinoma.

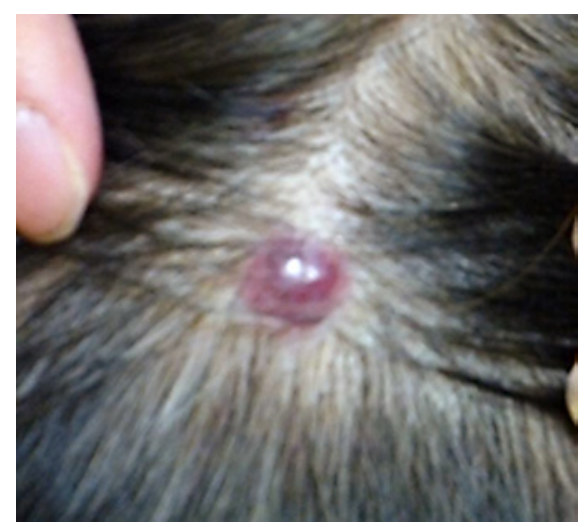

This examination is covered by Japanese medical insurance for cancer patients who experience progressive disease after all standard treatments. We decided to reintroduce lenvatinib while waiting for the results of this examination. The interval between the interruption of initial lenvatinib and its reintroduction was 7 months. Three months after the reintroduction of lenvatinib at $8 \mathrm{mg} /$ day, marked shrinkage of the tumor and a decrease in its contrast enhancement were seen (Fig. 1E). Serum Tg levels had also remarkably decreased in parallel with the treatment response following the reintroduction of lenvatinib. Grade 2 hypertension and grade 2 proteinuria were seen. Because the genomic profiling test revealed RET fusion, the patient stopped treatment with lenvatinib and entered a clinical trial of a selective RET inhibitor. 
Takinami and Yokota: Rechallenge with Lenvatinib for Metastatic Papillary Thyroid Carcinoma

\section{Discussion and Conclusion}

To the best of our knowledge, this is the first report of DTC that showed resensitization to rechallenge with lenvatinib after the development of refractoriness to initial lenvatinib and sorafenib. This case is clinically important because treatment options are limited for patients with DTC who progress despite receiving both lenvatinib and sorafenib.

"Drug resistance" is basically used as a synonym for progressive disease and "drug sensitization" refers to a response of a tumor. "Rechallenge" involves reintroduction of the same therapy after a drug holiday or intervening therapy. "Resensitization" is defined as a case of progressive disease after response to initial treatment followed by a subsequent tumor response upon drug rechallenge. In the current case, evaluations of tumor response were confirmed in the following three modalities. First, morphological alteration of the measurable tumor was evaluated in accordance with the Response Evaluation Criteria in Solid Tumors 1.1 (RECIST 1.1) [4], as was used in the phase III DECISION trial and SELECT trial, which revealed the efficacy of sorafenib and lenvatinib in DTC, respectively $[5,6]$. Second, intratumoral vascularity was additionally evaluated by contrast-enhanced CT because antiangiogenic targeted therapies provoke different carcinoma responses compared with conventional chemotherapy [7]. That is, a decrease in intratumoral enhancement suggests a tumor response to molecular-targeted therapies, even though tumor size does not change. Indeed, modified RECIST and Morphology, Attenuation, Size, and Structure (MASS) criteria, for which the contrast-enhanced area is measured, are applied to patients with hepatocellular carcinoma and renal cell carcinoma receiving antiangiogenic targeted therapies, respectively [8,9]. Third, the tumor marker of serum Tg level was measured because this level has been reported as a pharmacodynamic biomarker of DTC [5].

Tumor resensitization by rechallenge with TKIs has been reported in patients with renal cell carcinoma and non-small cell lung carcinoma [10]. One case study of DTC by Felicetti et al. [11] reported that a tumor initially responding to lenvatinib shrank upon rechallenge with lenvatinib after a 3-month interval. However, there are several differences in the treatment setting between this previous case and ours. First, CT images and serum Tg levels at the time of best response to initial lenvatinib were not available in this previous case. Therefore, it may be difficult to strictly evaluate progressive disease upon initial lenvatinib treatment. Second, the tumor size rapidly increased during a 3-month drug holiday, but showed a dramatic response to drug resumption. These findings raise the possibility that temporary interruption of lenvatinib treatment might have rapidly accelerated the cell growth of lenvatinib-sensitive clones. This phenomenon is known as a disease flare, as is occasionally experienced with TKIs [12]. A case of rechallenge with sorafenib was also reported in DTC [13]. In this case, new liver metastasis occurred after 8 months off sorafenib. However, rechallenge with sorafenib resulted in its complete disappearance. In these two previous reports on DTC, resensitization to lenvatinib or sorafenib after a drug holiday was discussed. However, the sensitivity to another TKI was not known in each case. In contrast, our case demonstrated resensitization by rechallenge with the initially administered TKI after the development of resistance to both lenvatinib and sorafenib.

The mechanisms through which resistance and resensitization to TKIs are acquired have remained incompletely understood. Although both lenvatinib and sorafenib are multitargeted TKIs, there are differences in the spectrum of targets between them. Lenvatinib predominantly inhibits pathways associated with vascular endothelial growth factor receptor (VEGFR) and fibroblast growth factor receptor (FGFR), whereas sorafenib targets Raf- 1 and BRAF at a low dose. These differences in targeted signaling pathway may play a role in the acquisition of resistance or resensitization. One hypothesis is that there is a mixture of lenvatinib-sensitiveand-resistant clones in tumors, and the proportions of clones change depending 
Takinami and Yokota: Rechallenge with Lenvatinib for Metastatic Papillary Thyroid Carcinoma

on the presence or absence of lenvatinib. Initial lenvatinib inhibits the growth of lenvatinibsensitive clones, inducing a clinical response. However, proportionally, lenvatinib-resistant clones then become dominant over sensitive clones, which causes tumor progression. Because lenvatinib is interrupted during sorafenib therapy, the regrowth of lenvatinib-sensitive clones is induced. Thus, rechallenge with lenvatinib may consequently be targeting lenvatinib-sensitive clones.

In our case, it should be considered that the dose of lenvatinib administered at rechallenge was higher than that at progressive disease during initial lenvatinib treatment. There is a possibility that the administration of a TKI produces an antitumor effect in a dosedependent manner because high-dose intermittent TKI improved the efficacy compared with a low dose [14].

Total thyroidectomy followed by RAI is generally performed to treat metastatic lesions, because metastases in DTC occasionally achieve partial or complete remission after surgery followed by RAI [15]. Unfortunately, total thyroidectomy followed by RAI was not a treatment option in this case, because the patient was unable to undergo total thyroidectomy due to severe local invasion even after tumor shrinkage upon treatment with lenvatinib. Furthermore, it is impossible to prescribe RAI to all patients with the indication of RAI, because facilities where RAI is available are limited in Japan.

In conclusion, tumors that are refractory to prior lenvatinib and sorafenib may be resensitized by rechallenge with lenvatinib in unresectable DTC. Therefore, rechallenge with lenvatinib may be one of the treatment options in patients with DTC who have experienced progressive disease after initial response to lenvatinib and subsequent sorafenib. Future prospective studies are required to investigate the efficacy of rechallenge with lenvatinib.

\section{Acknowledgements}

We would like to thank Editage (www.editage.com) for English language editing.

\section{Statement of Ethics}

The study protocol was approved by the Ethics Committee of the Shizuoka Cancer Center. The patient provided written informed consent for publication, including publication of the images.

\section{Disclosure Statement}

The authors declare no conflict of interest regarding this article.

\section{Funding Sources}

No funding was received for this research.

\section{Author Contributions}

All authors contributed equally to this work. 
Takinami and Yokota: Rechallenge with Lenvatinib for Metastatic Papillary Thyroid Carcinoma

\section{References}

1 Andresen NS, Buatti JM, Tewfik HH, Pagedar NA, Anderson CM, Watkins JM. Radioiodine ablation following thyroidectomy for differentiated thyroid cancer: literature review of utility, dose, and toxicity. Eur Thyroid J. 2017 Jul;6(4):187-96.

2 Li XY, Zhang B, Lin YS. The interpretation of 2015 American Thyroid Association management guidelines for adult patients with thyroid nodules and differentiated thyroid cancer [in Chinese]. Zhonghua Er Bi Yan Hou Tou Jing Wai Ke Za Zhi. 2017 Feb;52(4):309-15.

3 Filetti S, Durante C, Hartl D, Leboulleux S, Locati LD, Newbold K, et al. Thyroid cancer: ESMO Clinical Practice Guidelines for diagnosis, treatment and follow-up. Ann Oncol. 2019 Dec;30(12):1856-83.

4 Eisenhauer EA, Therasse P, Bogaerts J, Schwartz LH, Sargent D, Ford R, et al. New response evaluation criteria in solid tumours: revised RECIST guideline (version 1.1). Eur J Cancer. 2009 Jan;45(2):228-47.

5 Brose MS, Nutting CM, Jarzab B, Elisei R, Siena S, Bastholt L, et al. Sorafenib in radioactive iodine-refractory, locally advanced or metastatic differentiated thyroid cancer: a randomised, double-blind, phase 3 trial. Lancet. 2014 Jul;384(9940):319-28.

6 Schlumberger M, Tahara M, Wirth LJ, Robinson B, Brose MS, Elisei R, et al. Lenvatinib versus placebo in radioiodine-refractory thyroid cancer. N Engl J Med. 2015 Feb;372(7):621-30.

7 Tirumani SH, Fairchild A, Krajewski KM, Nishino M, Howard SA, Baheti AD, et al. Anti-VEGF molecular targeted therapies in common solid malignancies: comprehensive update for radiologists. Radiographics. 2015 MarApr;35(2):455-74.

8 Lencioni R, Llovet JM. Modified RECIST (mRECIST) assessment for hepatocellular carcinoma. Semin Liver Dis. 2010 Feb;30(1):52-60.

9 Smith AD, Shah SN, Rini BI, Lieber ML, Remer EM. Morphology, Attenuation, Size, and Structure (MASS) criteria: assessing response and predicting clinical outcome in metastatic renal cell carcinoma on antiangiogenic targeted therapy. AJR Am J Roentgenol. 2010 Jun;194(6):1470-8.

10 Kuczynski EA, Sargent DJ, Grothey A, Kerbel RS. Drug rechallenge and treatment beyond progression - implications for drug resistance. Nat Rev Clin Oncol. 2013 Oct;10(10):571-87.

11 Felicetti F, Nervo A, Piovesan A, Berardelli R, Marchisio F, Gallo M, et al. Tyrosine kinase inhibitors rechallenge in solid tumors: a review of literature and a case description with lenvatinib in thyroid cancer. Expert Rev Anticancer Ther. 2017 Dec;17(12):1093-8.

12 Chaft JE, Oxnard GR, Sima CS, Kris MG, Miller VA, Riely GJ. Disease flare after tyrosine kinase inhibitor discontinuation in patients with EGFR-mutant lung cancer and acquired resistance to erlotinib or gefitinib: implications for clinical trial design. Clin Cancer Res. 2011 Oct;17(19):6298-303.

13 Marotta V, Colao A, Faggiano A. Complete disappearance of liver metastases in a patient with iodine-refractory differentiated thyroid cancer subjected to sorafenib re-challenge. Endocrine. 2015 Dec;50(3):824-5.

14 Wang X, Zhang L, Goldberg SN, Bhasin M, Brown V, Alsop DC, et al. High dose intermittent sorafenib shows improved efficacy over conventional continuous dose in renal cell carcinoma. J Transl Med. 2011 Dec;9:220.

15 Song HJ, Qiu ZL, Shen CT, Wei WJ, Luo QY. Pulmonary metastases in differentiated thyroid cancer: efficacy of radioiodine therapy and prognostic factors. Eur J Endocrinol. 2015 Sep;173(3):399-408. 